This paper is published in the open archive of Mid Sweden University

DIVA http://miun.diva-portal.org

with permission of the publisher

Citation for the peer-reviewed published paper:

Edström P. Comparison of the DORT2002 Radiative Transfer Solution Method and the Kubelka-Munk Model. Nordic Pulp \& Paper Research Journal. $2004 ; 19(3): 397-403$.

URL to article at publishers site:

http://dx.doi.org/10.3183/NPPRJ-2004-19-03-p397-403 


\title{
Comparison of the DORT2002 radiative transfer solution method and the Kubelka-Munk model
}

\author{
Per Edström, Department of Technology, Physics and Mathematics, Mid Sweden University, Härnösand
}

KEYWORDS: Light scattering, Mathematical models, Radiative transfer, Kubelka-Munk, Light absorption, Errors, Reflectance calculations.

SUMMARY: The need for optical modeling of paper is obvious to provide connections between its optical response and the actual properties of the paper. It is argued that modern solution methods from radiative transfer theory could be considered instead of the Kubelka-Munk model, and a specific example, DORT2002, is tested. It is shown that Kubelka-Munk is a simple special case of DORT2002, and the two models and their coefficients are compared. A comprehensive list of advantages for the applied user of a model with higher dimensionality is supplied.

It is shown, by the use of DORT2002, that when the medium has finite thickness, the light distribution deviates from the perfectly diffuse even under the theoretically ideal conditions for which Kubelka-Munk was created. This effect causes errors in Kubelka-Munk reflectance calculations that may be up to $20 \%$ and more, even for a grammage of $80 \mathrm{~g} / \mathrm{m}^{2}$. The magnitude of the error shows a strong dependence on the degree of light absorption, with higher absorption giving greater error. DORT2002 can well be considered for increased understanding in cases where the level of accuracy of Kubelka-Munk reflectance calculations is not sufficient.

\section{ADDRESS OF THE AUTHOR: Per Edström}

(per.edstrom@mh.se): Department of Technology, Physics and Mathematics, Mid Sweden University, SE-871 88 Härnösand, Sweden.

\section{Introduction}

As is well known, the Kubelka-Munk light scattering and light absorption coefficients ( $s$ and $k$ ) are widely used in the pulp and paper industry in applications ranging from research projects to practical problems in paper mills. Examples are prediction of brightness and opacity of papers containing different pulps and fillers, or papers with multilayer structures (coated and printing papers). These coefficients provide a link between the (measured) reflectance factor, e.g. brightness, and properties of the paper sample, so that the reasons for a high or low reflectance may be better understood. The $s$ - and $k$-values are also linked to unit operations in pulp and paper technology through many investigations over the years. The reasons for this extensive use of the Kubelka-Munk model are most likely the simplicity of the equations (which was a major advantage before the introduction of personal computers) and the fact that they are invertible, roughly meaning that reflectance values can be calculated from $s$ and $k$, and that $s$ and $k$ can be calculated from reflectance values.

However, the Kubelka Munk equations are a special case of a solution method to what is known as the radiative transfer problem. Given the fast development of personal computers, it is worthwhile to investigate other solution methods that give a more detailed and more accurate description of the paper sample, and yet can easily be made accessible for the applied user.

Radiative transfer theory describes the interaction of radiation with scattering and absorbing media. It has been applied to such different applications as stellar atmospheres, infrared and visible light in space and the atmosphere, optical tomography and diffusion of neutrons. An industrially important application is light scattering in textile, paint, pigment films, paper and print, and accurate calculation methods are crucial for these sectors of industry.

Discrete ordinate solution methods for radiative transfer problems have been studied throughout the last century. The first approximate solution to the radiative transfer problem was presented by Schuster (1905), who considered radiation only in a forward and a backward direction. Clearly influenced by this, Kubelka and Munk (1931) developed their well-known model, which was further refined by Kubelka (1948; 1954). The models presented by Schuster and Kubelka and Munk, and others after them, are known as two-flux models.

By expressing an integral as a finite sum, what is now known as numerical quadrature, Wick (1943) gave the first general treatment of discrete ordinate methods for the radiative transfer problem. The terms in the sum can be interpreted as the contribution to flux or intensity from a discrete cone in spherical geometry. The polar angles of these cones are referred to as discrete ordinates, which has given the method its name, and the cones are called channels or streams. Using only two channels gives the earlier two-flux methods. If more channels are used, the methods are referred to as multi-flux methods or many-flux methods. Chandrasekhar (1944a) described a method using spherical harmonics, but having read Wick's article, he adopted the discrete ordinate method, and further refined it (1944b). Later, he wrote a classic exposition on radiative transfer theory in book form (Chandrasekhar 1960), and since then the area has expanded tremendously.

Along with this expansion, there has been a continuous development of solution methods for the radiative transfer problem. When Kubelka and Munk presented their model, it was state-of-the-art, but now it should be seen as the approximation it is. Several limitations for the Kubelka-Munk model have been reported, for example concerning dependencies between the $s$ and $k$ coefficients for translucent or strongly absorbing media (Foote 1939; Nordman et al. 1966; Moldenius 1983; Rundlöf and Bristow 1997), and attempts have been made to attribute some of this behavior to intrinsic errors of - or pheno- 
mena not included in - the Kubelka-Munk model (van den Akker 1966; van den Akker 1968; Koukoulas and Jordan 1997; Granberg and Edström 2003). Despite these limitations, the Kubelka-Munk model is in widespread use for multiple scattering calculations in paper, paper coatings, printed paper, paint, plastic and textile, probably due to its explicit form and ease of use. These are also reasons for the continued usage where the accuracy is sufficient, and where there are no reported limitations. However, new solution methods with better accuracy and a larger range of applicability should be considered in many cases.

The DORT2002 software is a fast and accurate tool for solving radiative transfer problems in vertically inhomogeneous turbid media, using a discrete ordinate model geometry. DORT2002 is implemented in MATLAB, and is adapted to light scattering simulations in paper and print. The model was presented in a recent paper by Edström (2003), which thoroughly describes the model and its features. Accuracy and numerical performance of DORT2002 have been evaluated by Edström (2004), and the solution method was found to be accurate, very fast, and numerically stable.

The purpose of this paper is to draw attention to the potential of models of higher dimensionality, for example those provided by radiative transfer theory, since modern computers make it fully tractable to use such models. In this paper, DORT2002 is used as an example of such models. Section 2 of this paper gives a short outline of a radiative transfer formulation of the light scattering problem, and in section 3 a translation between the coefficients of the Kubelka-Munk and DORT2002 models is given, together with a discussion of when the translation is valid. Section 4 covers the application of KubelkaMunk and DORT2002 to a number of test problems, provides and discusses important numerical results, and specifically notes that Kubelka-Munk may fail to provide correct results even under ideal conditions. Section 5 supplies a list of advantages for the applied user of a model with higher dimensionality, and notes that the angular resolution of DORT2002 enables it to exceed Kubelka-Munk extensively by giving a more detailed and more accurate description of the paper sample. Finally, some concluding discussions and references are given.

\section{The physical problem}

For an ideally reflecting medium, all incoming light is specularly reflected at the surface. For a turbid medium, transmission as well as absorption and multiple scattering inside the medium have to be taken into consideration. In this paper, the problem is studied in plane-parallel geometry, where the horizontal extension of the medium is assumed to be large enough to give no boundary effects at the sides. The boundary conditions at the top and bottom boundary surfaces, including illumination, are assumed to be time- and space-independent on the respective boundary surface. The radiation is assumed to be monochromatic, or confined to a sufficiently narrow wavelength range to make scattering and absorption constant. The scattering is assumed to be conservative, i.e. without change in frequency between incoming and outgoing radiation. The medium is treated as a continuum of scattering and absorption sites. Kubelka-Munk and DORT2002 both use these same assumptions about the medium. The main difference is that Kubelka-Munk is limited to diffuse illumination and scattering, while DORT2002 handles any illumination and any scattering thanks to the angle-resolved model geometry. DORT2002 provides this resolution by dividing the space into any number of "channels", corresponding to different directions, while Kubelka-Munk only considers the averaged directions "up" and "down".

The energy flow is thought of as non-interacting beams of radiation in all directions. This makes it possible to treat the beams separately. The intensity, $I$, of the radiation is always considered to be positive. When radiation traverses the infinitesimal thickness $d s$ of the medium in its direction of propagation, a fraction is extinct due to absorption and scattering. The intensity then becomes $I+d I$, and the extinction coefficient is defined as $\sigma_{e}=-\frac{d I}{I d s}$. The extinction coefficient can be separated into two parts, called the absorption and scattering coefficients, $\sigma_{a}$ and $\sigma_{s}$, corresponding to the two different origins of the extinction. They are related to the extinction coefficient through $\sigma_{e}=\sigma_{a}+\sigma_{s}$. It should be noted that these coefficients are real physical quantities, and not defined through a model equation such as the Kubelka-Munk $s$ and $k$. A convenient measure is the single scattering albedo, which is the probability for scattering given an extinction event, and is defined as $a=\sigma_{s} / \sigma_{e}=\sigma_{s} /\left(\sigma_{a}+\sigma_{s}\right)$.

The phase function, $p$, specifies the angular distribution of the scattered radiation. If the phase function is normalized by $\int_{0}^{2 \pi} \int_{0}^{\pi} \sin \theta \frac{p\left(\theta^{\prime}, \varphi^{\prime} ; \theta, \varphi\right)}{4 \pi} d \theta d \varphi=1$

where $\theta$ and $\varphi$ are the polar and azimuthal angle coordinates of spherical geometry for the direction of the radiation (in the remainder of this paper, primed arguments correspond to incident radiation), this can be given a probabilistic interpretation. Given that radiation in the direction $\left(\theta^{\prime}, \varphi^{\prime}\right)$ is scattered, the probability that it is scattered into the cone of solid angle $d \theta d \varphi$ centered on the direction $(\theta, \varphi)$ is

$$
\frac{p\left(\theta^{\prime}, \varphi^{\prime} ; \theta, \varphi\right) d \theta d \varphi}{4 \pi} .
$$

It is common that the shape of the scattering probabilitydistribution is controlled by a parameter in the phase function, an asymmetry factor.

For plane-parallel geometry, it is convenient to measure distances normal to the surface of the medium. This coincides with the $z$-axis in a Cartesian coordinate system if the surface is placed in the $x-y$-plane, and it is evident that $d z=d s \cos \theta$. The optical depth, measured from the top surface and down, is then defined as $\tau(z)=\int_{z}^{\infty} \sigma_{e} d z^{\prime}$.

It is also common to introduce $\mathrm{u}=\cos \theta$ (and sometimes $\mu=|\cos \theta|)$, which gives $d \tau=-\sigma_{\mathrm{e}} u d s$. Chandrasekhar (1960, eq. I.71) states the equation of radiative transfer 
for a scattering plane-parallel medium as

$u \frac{d I(\tau, u, \varphi)}{d \tau}=I(\tau, u, \varphi)-\frac{a}{4 \pi} \int_{0}^{2 \pi} \int_{-1}^{1} p\left(u^{\prime}, \varphi^{\prime} ; u, \varphi\right) I\left(\tau, u^{\prime}, \varphi^{\prime}\right) d u^{\prime} d \varphi^{\prime}$.

The integral term on the right hand side is a source function. It gives the intensity scattered from all incoming directions at a point to a specified direction.

\section{A comparison between the coefficients of the Kubelka-Munk and DORT2002 models}

The aim of this section is to make a comparison of the scattering and absorption coefficients $s$ and $k$ of the Kubelka-Munk model, and the real physical quantities $\sigma_{s}$ and $\sigma_{a}$ - also known as cross sections - used in radiative transfer, and to specify under what conditions an exact translation is valid. Since the coefficients of the KubelkaMunk model are so well known and in such widespread use, a translation between these is valuable for the future use of both model types.

The Kubelka-Munk equations can be written

$$
\left\{\begin{array}{l}
-d i=-(s+k) i d x+s j d x \\
d j=-(s+k) j d x+s i d x
\end{array},\right.
$$

where $i$ is the intensity in the downward direction and $j$ is the intensity in the upward direction. $s$ and $k$ are the light scattering and absorption coefficients, and $x$ is the distance measured from the background and upwards. This can be rewritten as

$$
\left\{\begin{array}{l}
-\frac{d i}{d x}=-k i-s i+s j \\
\frac{d j}{d x}=-k j-s j+s i
\end{array} .\right.
$$

The first term on the right hand side is absorption, i.e. the intensity "disappears", the second term is intensity scattered into the opposite direction, and the third term is a contribution to the intensity, scattered from the opposite direction. The derivation of the Kubelka-Munk equations assumes that the light is perfectly diffuse, but the fact that light incident at an angle has a longer optical path to a certain depth in the medium is not explicitly considered (or it can be assumed to be built into the coefficients $s$ and $k$ ). Hence, $k$ is the part of the intensity that is absorbed and "disappears" upon passage of the infinitesimal thickness $d x$ of the medium. $s$ is the part of the intensity that is scattered to the opposite direction upon passage of the infinitesimal thickness $d x$ of the medium.

As mentioned previously, the DORT2002 model is a more general model than Kubelka-Munk. In the simple special case with only two channels, corresponding to the only two directions "up" and "down" of Kubelka-Munk, the DORT2002 equations can be rewritten as

$$
\left\{\begin{array}{l}
-\mu \frac{d i(\tau, \mu)}{d \tau}=i(\tau, \mu)-\frac{1}{2} \frac{\sigma_{s}}{\sigma_{a}+\sigma_{s}} \int_{0}^{1} j\left(\tau, \mu^{\prime}\right) d \mu^{\prime}-\frac{1}{2} \frac{\sigma_{s}}{\sigma_{a}+\sigma_{s}} \int_{0}^{1} i\left(\tau, \mu^{\prime}\right) d \mu^{\prime}, \\
\mu \frac{d j(\tau, \mu)}{d \tau}=j(\tau, \mu)-\frac{1}{2} \frac{\sigma_{s}}{\sigma_{a}+\sigma_{s}} \int_{0}^{1} j\left(\tau, \mu^{\prime}\right) d \mu^{\prime}-\frac{1}{2} \frac{\sigma_{s}}{\sigma_{a}+\sigma_{s}} \int_{0}^{1} i\left(\tau, \mu^{\prime}\right) d \mu^{\prime}
\end{array}\right.
$$

where $i$ and $j$ generally depend on $\tau$ and $\mu$. In the discrete ordinate approximation (using Double-Gauss numerical quadrature) for two channels, $i(\tau, \mu)$ and $j(\tau, \mu)$ are replaced with their hemispherical averages, $i(\tau)$ and $j(\tau)$. For diffuse light, the average of $\mu$ becomes

$$
\int_{0}^{1} \mu d \mu=\frac{1}{2} \text {. }
$$

It should be noted here, that the distance $x$ used in Kubelka-Munk, and the optical depth $\tau$ used in DORT2002 are in opposite directions. Therefore, $d \tau=-\sigma_{e}$ $d x=-\left(\sigma_{a}+\sigma_{s}\right) d x$, and the DORT2002 equations become

$$
\left\{\begin{array}{l}
-\frac{d i}{d x}=-2 \sigma_{a} i-2 \sigma_{s} i+\sigma_{s} i+\sigma_{s} j \\
\frac{d j}{d x}=-2 \sigma_{a} j-2 \sigma_{s} j+\sigma_{s} j+\sigma_{s} i
\end{array} .\right.
$$

Here the equations have been written in this form for ease of comparison. The first term on the right hand side is absorption, i.e. the intensity "disappears", and the second term is totally scattered intensity. The third term is a contribution to the intensity, "scattered" from the same direction, and the fourth term is a contribution to the intensity, scattered from the opposite direction. Together the second and third terms form the net scattered intensity to the opposite direction. The fact that light incident at an angle has a longer optical path to a certain depth in the medium is explicitly considered, and the effect is the factor $1 / 2$ from the average of $\mu$. Hence, $2 \sigma_{a}$ is the part of the intensity that is absorbed and "disappears" upon passage of the infinitesimal thickness $d x$ of the medium. $\sigma_{s}$ is the part of the intensity that is scattered to the opposite direction upon passage of the infinitesimal thickness $\mathrm{dx}$ of the medium.

The conditions, under which an exact translation between the coefficients of the Kubelka-Munk and the DORT2002 models is valid, are: perfectly diffuse light, perfectly isotropic scattering, and only two channels in the DORT2002 model. Under these conditions it is obvious from the calculations above that the following relations hold: $k=2 \sigma_{a}$ and $s=\sigma_{s}$.

The three validity conditions each deserve a short discussion. First, if the light has an angular distribution other than perfectly diffuse, the average of $\mu$ is changed. Thus $E q$ (5) is not valid, and $E q$ (4) does not yield $E q(6)$. Second, if the scattering is anisotropic, the phase function is no longer $\equiv 1$, and the integrals in $E q$ (4), where the phase function is implicitly present, are changed so that they are no longer the hemispherical average. Thus, $E q$ (4) does not yield $E q(6)$. The third validity condition, which is an effect of the finite thickness of the medium, deserves a longer discussion, which is contained in the section on application tests. Here it can simply be noted that in the case of more than two channels, $E q$ (4) becomes the far more complicated $E q(1)$, and a simple translation to $E q(6)$ is not possible.

There remains one more comment to give on coefficients at this point; van den Akker (1949) showed that the Kubelka-Munk differential equations remain unchanged if the original scattering and absorption coefficients $S$ 
and $K$ (unit $\mathrm{m}^{-1}$ ) are replaced by the specific scattering and absorption coefficients $s$ and $k$ (unit $\mathrm{m}^{2} \cdot \mathrm{kg}^{-1}$ ), and the thickness $X$ is replaced by weight per unit area (grammage) $W$. He proposed that these should be used instead, based on the fact that in practical application of the Kubelka-Munk model, the thickness of a paper may change significantly without affecting the reflectance, suggesting that the light scattering remains unchanged, which naturally is also true for the grammage. The use of grammage is now common practice in paper-related applications. All relationships are unaffected by this. This same convention is readily applied to the DORT2002 model. Although derived for scattering and absorption coefficients with unit $\mathrm{m}^{-1}$ and optical depth $\tau$, the equations remain unchanged if specific scattering and absorption coefficients with unit $\mathrm{m}^{2} \cdot \mathrm{kg}^{-1}$ are used instead, together with the grammage $W$. As for the Kubelka-Munk model, all relationships are unaffected by this. It should be noted that for both Kubelka-Munk and DORT2002, this can only be done if the scattering and absorption coefficients as well as the density of the medium are constants. Otherwise the specific scattering and absorption coefficients as well as the grammage will not be constants, which is assumed by the theory.

\section{Application tests of the Kubelka-Munk and DORT2002 models}

The two-channel case

Theoretically, Kubelka-Munk is the simple two channel special case for DORT2002 if illumination, phase function and underlying surface are perfectly diffuse. Therefore, DORT2002 should yield results identical to Kubelka-Munk under these conditions. This was tested for various scattering and absorption coefficients and different thicknesses/grammages by calculating total reflectance for a medium over a black background, $R_{0}$, and over an opaque pad of the medium itself, $R_{\infty}$. The Kubelka-Munk calculations were done according to the equations in Pauler (2002). The definitions for the scattering and absorption coefficients differ between Kubelka-Munk ( $s$ and $k$ ) and DORT2002 $\left(\sigma_{s}\right.$ and $\sigma_{a}$ ), so the relations given in the previous section were used for translation. Table 1 gives the parameter values used, and all combinations were tested, giving a total of 27 test cases. The parameter values were chosen so that the combinations would represent a range of paper qualities, both unprinted, dyed and printed.

\begin{tabular}{llll} 
Table 1. Parameters for the test cases. & \\
\hline Grammage $\left[\mathrm{g} / \mathrm{m}^{2}\right]$ & $\mathrm{w} 1=80$ & $\mathrm{w} 2=50$ & $\mathrm{w} 3=30$ \\
Scattering $\left[\mathrm{m}^{2} / \mathrm{kg}\right]$ & $\mathrm{s} 1=20$ & $\mathrm{~s} 2=40$ & $\mathrm{~s} 3=80$ \\
Absorption $\left[\mathrm{m}^{2} / \mathrm{kg}\right]$ & $\mathrm{k} 1=0.1$ & $\mathrm{k} 2=1.0$ & $\mathrm{k} 3=100$
\end{tabular}

In all 27 test cases, Kubelka-Munk gave identical results to DORT2002, when only two channels were used, as should be expected. This verifies that DORT2002 indeed becomes Kubelka-Munk when using only two channels.
The multi-channel case:

Effects of finite medium thickness

It is now tempting to think that if illumination, phase function and underlying surface were perfectly diffuse, the resulting light distribution would be perfectly diffuse. There would therefore be no need for DORT2002 in this case at all, since with two channels it gives identical results to Kubelka-Munk. However, this is wrong. The light distribution deviates from the perfectly diffuse due to the finite thickness of the medium, since some light escapes through the lower boundary. With more channels, say twenty, DORT2002 detects and quantifies this.

It is important to realize that scattering is a local phenomenon, and even if every scattering event is perfectly diffuse, the total scattered light distribution needs not be perfectly diffuse due to edge effects. Kubelka and Munk did not recognize this fact in the first paper on their model. They assume infinite horizontal extension to avoid edge effects, but do not consider edge effects due to finite thickness. Even the follow-up paper by Kubelka (1948), that aims to theoretically derive a range of validity for that model, does not recognize this fact but rather assumes the opposite line of reasoning: "Practically it will be so when the illumination is a perfectly diffuse one and when the material forming specimens of different thickness reflects and transmits always perfectly diffused light only". This is an intuitively tempting belief, but it is not true when the medium has finite thickness since some light escapes through the lower boundary, and the light distribution becomes slightly changed. Kubelka (1948) defines $u d x$ and $v d x$ as the average path of the light passing through the layer $d x$ going down and up, respectively, taking into consideration the various directions of the beams of radiation. Kubelka is right when he states that the model is valid when $u=v=$ const, but wrong when he assumes that $u=v=$ const if scattering and illumination are perfectly diffuse - it will still not be true for a medium of finite thickness.

This effect is far from negligible, and can sometimes be very large indeed. This is illustrated by Table 2, which presents the results of the same 27 test cases as in the previous section, but now using twenty channels in DORT2002. This can be considered to be a true value, since simple tests show that DORT2002 has already converged (it has been shown by Edström (2004) that DORT2002 converges to the true value when the number of channels increases).

From Table 2 it is clear that the magnitude of the relative error shows a strong dependence on the degree of absorption, with higher absorption giving greater relative error. There is also a weak dependence on the degree of scattering, higher scattering giving somewhat smaller relative error. This is in accordance with earlier papers that address problems when using Kubelka-Munk on translucent or strongly absorbing media (Foote 1939; Nordman et al. 1966; Moldenius 1983; Rundlöf and Bristow 1997). Thus, DORT2002 offers a partial explanation to these previously reported problems, as it quantifies the deviation from perfectly diffuse light distribution 
due to finite medium thickness.

As can also be seen, the relative difference between the erroneous total reflectance given by Kubelka-Munk and the true value given by DORT2002 can be up to $20 \%$ and more, depending on the properties of the medium, even under the theoretically ideal conditions for which Kubelka-Munk was created. It should be noted that the Kubelka-Munk model causes these very large errors even for a grammage of $80 \mathrm{~g} / \mathrm{m}^{2}$. However, Kubelka-Munk is normally used in a self-consistent manner, i.e. values of $s$ and $k$ are normally determined from measured $R_{0}$ and $R_{\infty}$ for samples of a certain grammage, and then, using these $s$ and $k$ values, $R_{0}$ and $R_{\infty}$ for a mixture (often of the same total grammage) of the samples is predicted. This selfconsistent usage makes the errors cancel in varying degree, and they will not always be apparent. But if these $s$ and $k$ values are applied to a sample of different total grammage, the errors will start to be visible.

Cases with large errors, as in the tests above, are not infrequent in practice. On the contrary, there are low opacity papers that are translucent, and there are heavily dyed papers that are strongly absorbing, not to mention full tone prints with inks that have very strong light absorption in a certain range. It remains to be seen what this implies for the application of the Kubelka-Munk model.

Table 2. Results of the tests.

\begin{tabular}{|c|c|c|c|c|c|c|}
\hline \multirow[b]{2}{*}{ Case } & \multicolumn{3}{|c|}{$\mathbf{R}_{\infty}$} & \multicolumn{3}{|c|}{$\mathrm{R}_{0}$} \\
\hline & $\begin{array}{l}\text { Kubelka- } \\
\text { Munk }\end{array}$ & $\begin{array}{l}\text { DORT2002, } \\
20 \text { channels }\end{array}$ & $\begin{array}{l}\text { Relative } \\
\text { Difference }\end{array}$ & $\begin{array}{l}\text { Kubelka- } \\
\text { Munk }\end{array}$ & $\begin{array}{l}\text { DORT2002, } \\
20 \text { channels }\end{array}$ & $\begin{array}{c}\text { Relative } \\
\text { Difference }\end{array}$ \\
\hline $\mathrm{w} 1, \mathrm{~s} 1, \mathrm{k} 1$ & 0,8682 & 0,8502 & $2,12 \%$ & 0,6077 & 0,5503 & $10,43 \%$ \\
\hline w1,s1,k2 & 0,6417 & 0,6043 & $6,19 \%$ & 0,5455 & 0,4899 & $11,35 \%$ \\
\hline w1,s1,k3 & 0,0455 & 0,0377 & $20,69 \%$ & 0,0455 & 0,0377 & $20,69 \%$ \\
\hline w1,s2,k1 & 0,9049 & 0,8914 & $1,51 \%$ & 0,7529 & 0,7027 & $7,14 \%$ \\
\hline w1,s2,k2 & 0,7298 & 0,6981 & $4,54 \%$ & 0,6827 & 0,6340 & $7,68 \%$ \\
\hline w1,s2,k3 & 0,0839 & 0,0702 & $19,52 \%$ & 0,0839 & 0,0702 & $19,52 \%$ \\
\hline w1,s3,k1 & 0,9317 & 0,9218 & $1,07 \%$ & 0,8552 & 0,8200 & $4,29 \%$ \\
\hline w1,s3,k2 & 0,8000 & 0,7745 & $3,29 \%$ & 0,7832 & 0,7481 & $4,69 \%$ \\
\hline w1,s3,k3 & 0,1459 & 0,1239 & $17,76 \%$ & 0,1459 & 0,1239 & $17,76 \%$ \\
\hline w2,s1,k1 & 0,8682 & 0,8502 & $2,12 \%$ & 0,4959 & 0,4424 & $12,09 \%$ \\
\hline w2,s1,k2 & 0,6417 & 0,6043 & $6,19 \%$ & 0,4610 & 0,4077 & $13,07 \%$ \\
\hline w2,s1,k3 & 0,0455 & 0,0377 & $20,69 \%$ & 0,0455 & 0,0377 & $20,69 \%$ \\
\hline w2,s2,k1 & 0,9049 & 0,8914 & $1,51 \%$ & 0,6615 & 0,6049 & $9,36 \%$ \\
\hline w2,s2,k2 & 0,7298 & 0,6981 & $4,54 \%$ & 0,6186 & 0,5631 & $9,86 \%$ \\
\hline w2,s2,k3 & 0,0839 & 0,0702 & $19,52 \%$ & 0,0839 & 0,0702 & $19,52 \%$ \\
\hline w2,s3,k1 & 0,9317 & 0,9218 & $1,07 \%$ & 0,7942 & 0,7484 & $6,12 \%$ \\
\hline w2,s3,k2 & 0,8000 & 0,7745 & $3,29 \%$ & 0,7468 & 0,7018 & $6,41 \%$ \\
\hline w2,s3,k3 & 0,1459 & 0,1239 & $17,76 \%$ & 0,1459 & 0,1239 & $17,76 \%$ \\
\hline w3,s1,k1 & 0,8682 & 0,8502 & $2,12 \%$ & 0,3730 & 0,3306 & $12,83 \%$ \\
\hline w3,s1,k2 & 0,6417 & 0,6043 & $6,19 \%$ & 0,3561 & 0,3128 & $13,84 \%$ \\
\hline w3,s1,k3 & 0,0455 & 0,0377 & $20,69 \%$ & 0,0455 & 0,0377 & $20,69 \%$ \\
\hline w3,s2,k1 & 0,9049 & 0,8914 & $1,51 \%$ & 0,5428 & 0,4870 & $11,46 \%$ \\
\hline w3,s2,k2 & 0,7298 & 0,6981 & $4,54 \%$ & 0,5198 & 0,4641 & $12,00 \%$ \\
\hline w3,s2,k3 & 0,0839 & 0,0702 & $19,52 \%$ & 0,0839 & 0,0702 & $19,52 \%$ \\
\hline w3,s3,k1 & 0,9317 & 0,9218 & $1,07 \%$ & 0,7027 & 0,6478 & $8,47 \%$ \\
\hline w3,s3,k2 & 0,8000 & 0,7745 & $3,29 \%$ & 0,6750 & 0,6208 & $8,73 \%$ \\
\hline w3,s3,k3 & 0,1459 & 0,1239 & $17,76 \%$ & 0,1459 & 0,1239 & $17,76 \%$ \\
\hline
\end{tabular}

\section{Advantages of higher dimensionality}

Two-flux models, such as Kubelka-Munk, are inherently one-dimensional, which of course is a reason for the relative simplicity and ease of use. But this comes at the cost of the inability to model processes in higher dimensions, such as scattering. Radiative transfer is a fully three-dimensional theory, which means that many physical processes can be modeled without approximations.

As mentioned earlier, one can see DORT2002 as a natural generalization of the Kubelka-Munk model, or, equivalently, one can see Kubelka-Munk as a simple special case of DORT2002. More specifically, KubelkaMunk is exactly the special case of DORT2002 where the illumination and scattering are completely isotropic, and where only two channels are considered. Therefore, all previous knowledge can still be used, as well as all tables, parameter values, measurements, instruments etc, so nothing needs to be discarded. However, there may be much to gain from a fully three-dimensional model.

The angular resolution of DORT2002, which is possible thanks to the higher dimensionality, enables it to exceed Kubelka-Munk in many ways, as indicated by the following list.

- An angle-resolved model can simulate the angular distribution of the reflected and transmitted light, while Kubelka-Munk only handles the perfectly diffuse case. Even this is handled more or less erroneously, since the fact that the light distribution deviates from the perfectly diffuse due to the finite thickness of the medium is ignored. DORT2002 is not limited to diffuse scattering, but can handle any asymmetric scattering as well. This makes it possible to model the scattering more accurately, since most media scatter more or less asymmetrically. Under practical viewing conditions, illumination resembling the perfectly diffuse case is probably rare. DORT2002 may be used to describe those cases and provide new insight into the connections between the properties of the paper and its perceived quality.

- Different illumination and detection geometries, e.g. combinations of collimated sources and diffuse light with asymmetric distribution, can be handled by DORT2002, while Kubelka-Munk only handles the perfectly diffuse case. For example, this makes it possible to use laser beams in measurements, and integrating spheres are not needed. This case is often highly asymmetric as shown by measurements reported by Granberg (2003).

- An angle-resolved model can simulate instrument geometry, and can take into consideration the deviation from perfectly diffuse conditions which are imposed by the instrument itself, i.e. from openings, gloss trap etc. While Kubelka-Munk assumes perfect conditions, DORT2002 can quantify the error and correct for it. It is also possible to calculate error corrections for different instrument geometries beforehand, to allow the instruments to correct their own errors.

- Different instrument geometries (e.g. d/0 and 45/0) rank the same samples differently (Popson and Malthouse 1996), which can be explained by DORT2002 but not by Kubelka-Munk. This can make it easier to understand and communicate measurements and calibration between different instrument 
geometries, but also between sectors of industry with different standards.

- Intrinsic errors of the Kubelka-Munk model, such as apparent parameter dependencies for translucent or strongly absorbing media, can be identified and quantified by DORT2002. This was recently done in a paper by Granberg and Edström (2003). An accurate characterization of the amount of chromophoric material absorbing strongly in the UV-region of the spectrum is useful for example in studies of the yellowing of wood pulp.

- DORT2002 has an open and modularized structure, which makes it possible to easily add functions for handling more phenomena. For example, DORT2002 is already prepared to handle refraction between layers with different index of refraction, to be combined with surface models to handle gloss, and to handle fluorescence, which is needed to simulate media with optical brightening agents and calculate whiteness and brightness. All of these examples are of major interest in the development of printing papers, where a better understanding of the connections between the properties of the paper and its perceived optical quality is clearly needed. Needless to say, the possibility of including surface properties quite dramatically increases the usefulness of optical modeling, since gloss is often discussed in connection with printed images, see Béland (2001) and references therein. Kubelka-Munk handles none of the specified examples, since it has a closed structure that does not allow for new phenomena.

\section{Discussion}

There is an obvious need for optical modeling in the paper industry for printing papers with increased demands for the "right appearance". This need is also driven by increasing competition from other media. The modeling would provide connections between the actual properties of the paper products and their perceived optical quality. Indeed, the appearance of paper products is also becoming increasingly important in the field of packaging and hygiene products, where the "right impression" also includes the appearance of the product.

Examples of areas where optical modeling is used within the paper industry are fine-tuning the papermaking process, designing new paper qualities, color management from pre-press to print, and evaluating printing techniques. Today the Kubelka-Munk model (or extended models thereof) is most widely used to cover these applications. It has been argued here that in many cases modern solution methods from radiative transfer theory could be considered for increased understanding, and a specific example, DORT2002, has been suggested and tested.

The conditions, under which an exact translation between the coefficients of the Kubelka-Munk and the DORT2002 models is valid, have been shown to be: perfectly diffuse light, perfectly isotropic scattering, and only two channels in the DORT2002 model. Under these conditions it has been shown to hold that $s=\sigma_{s}$ and $k=2 \sigma_{a}$

It has been shown that when the medium has finite thickness, the light distribution deviates from the perfectly diffuse even if illumination and scattering are perfectly diffuse, which is in opposition to what one would intuitively expect. This effect is caused by light escaping through the lower boundary of the medium, and causes errors in Kubelka-Munk reflectance calculations that can be up to $20 \%$ and more, even for a grammage of $80 \mathrm{~g} / \mathrm{m}^{2}$. The magnitude of the error shows a strong dependence on the degree of absorption, with higher absorption giving greater error. This confirms previously reported problems with Kubelka-Munk for strongly absorbing media, and DORT2002 offers a partial explanation of these problems, as it can describe this effect and quantify the Kubelka-Munk errors. Cases with large errors are not infrequent in practice, but include cases such as heavily dyed papers or full tone prints. Further investigation is needed to establish what this implies for the application of the Kubelka-Munk model.

Kubelka-Munk is normally used in a self-consistent manner, i.e. $s$ and $k$ values are determined from measured $R_{0}$ and $\mathrm{R}_{\infty}$ for samples of a certain grammage, and then, using these $s$ and $k$ values, $R_{0}$ and $\mathrm{R}_{\infty}$ for a mixture often of the same total grammage - of the samples is predicted. The errors will therefore cancel in varying degree and will not always be apparent. But if these $s$ and $k$ values are applied to a sample of different total grammage, the errors will begin to be visible. The errors will also become noticeable if more correct $s$ and $k$ values are obtained by other means, and then used to predict $R_{0}$ and $\mathrm{R}_{\infty}$. Conversely, if Kubelka-Munk is used to determine $s$ and $k$ values for a sample where the effect of finite thickness is large, the $s$ and $k$ values will also contain these errors, which will be carried over to a more accurate model if it uses these $s$ and $k$ values. This means that the more accurate model needs to have its own solution procedure to determine its scattering and absorption parameters. This amounts to an inverse problem, for which a solution procedure for DORT2002 is under development.

From the point of view of the applied user, angle-resolved models such as DORT2002 have several advantages compared to the Kubelka-Munk model. The angular distribution of reflection and transmission may be modeled, as well as different scattering asymmetries of the bulk. Collimated light can be used to analyze the optical response of a sample, while the Kubelka-Munk model is limited to diffuse light. Since any illumination and detection conditions may be handled, the interior of instruments otherwise closed for inspection can be simulated, and the influence of instrument geometry on measurements may be evaluated. This makes it possible to suggest measurement corrections for deviations due to instrument geometry, and to make calibration and measurements with different instrument geometries comparable. Furthermore, DORT2002 is consistent for translucent and highly absorbing media, and it is prepared to be combined with a surface model to handle 
gloss. It is also prepared for a future implementation of fluorescence, which will allow the effect of OBA (optical brightening agents) in paper and print to be modeled. This opens the field for finding new connections between the properties of paper and its perceived optical quality. The whiteness and brightness of paper cannot be designed with the Kubelka-Munk model since fluorescence phenomena are not explicitly included.

These improvements are important for a number of reasons. Paper may be translucent, glossy and strongly absorbing, e.g. low opacity paper, calendered paper, and heavily dyed paper or full tone prints. The standardized measurement geometries (d/0 and 45/0) for reflectance factors such as brightness of paper may give different rankings, which cannot be explained by the KubelkaMunk model, but is readily given by DORT2002. Moreover, there is experimental evidence that the reflection and transmission of paper and print deviates from the Kubelka-Munk model description (Granberg (2003)), which can be interpreted more accurately by DORT2002.

As mentioned earlier, since Kubelka-Munk is a simple special case of DORT2002, all previous knowledge such as tables, parameter values, measurements etc can still be used, so nothing needs to be discarded. On the contrary, it provides a strong foundation for future work with both models. A solution procedure for the inverse problem of DORT2002 is required to complete the picture, but DORT2002 may well be considered at present for increased understanding in cases where the level of accuracy of Kubelka-Munk reflectance calculations is not acceptable.

\section{Acknowledgements}

The author wishes to thank professor Tetsu Uesaka for valuable comments on the manuscript and for discussions on applied paper optics. Thanks also go to Dr Mats Rundlöf and Dr Hjalmar Granberg. This work was financially supported by the Swedish printing research program T2F, "TryckTeknisk Forskning", which is gratefully acknowledged.

\section{Literature}

van den Akker, J. A. (1949): Scattering and Absorption of Light in Paper and Other Diffusing Media, TAPPI 32, pp 498-501.

van den Akker, J. A. (1966): Discussion on Relationships Between Mechanical and Optical Properties of Paper Affected by Web Consolidation, Trans. Symp. Consolidation Paper Web, Tech. Sect. Brit. Paper Board Maker's Assoc., London, UK, pp 948-950 van den Akker, J. A. (1968): Theory of Some of the Discrepancies Observed in Application of the Kubelka-Munk Equations to Particulate Systems, in "Modern Aspects of Reflectance Spectroscopy", W. W. Wendlandt, Ed., Plenum Press, New York, NY, USA, pp 27-46.

Béland, M.-C. (2001): Gloss Variation of Printed Paper: Relationship Between Topography and Light Scattering, Doctoral Thesis, Royal Institute of Technology, Stockholm.

Chandrasekhar, S. (1944a): On the Radiative Equilibrium of a Stellar Atmosphere, Astrophys. J. 99, pp 180-190.

Chandrasekhar, S. (1944b): On the Radiative Equilibrium of a Stellar Atmosphere II, Astrophys. J. 100, pp 76-86.

Chandrasekhar, S. (1960): Radiative Transfer, Dover, New York.

Edström, P. (2003): A Fast and Stable Solution Method for the Radiative Transfer Problem, to appear in SIAM Rev.

Edström, P. (2004): Numerical Performance of the DORT2002 Radiative Transfer Solution Method, submitted to Appl. Numer. Math.

Foote, W. J. (1939): An Investigation of the Fundamental Scattering and Absorption Coefficients of Dyed Handsheets, Paper Trade J. 109, pp 333-340.

Granberg, H. (2003): Optical Response from Paper: Angle-dependent Light Scattering Measurements, Modelling and Analysis, Doctoral Thesis, Royal Institute of Technology, Stockholm.

Granberg, H. and Edström, P. (2003): Quantification of the Intrinsic Error of the Kubelka-Munk Model Caused by Strong Light Absorption, J. Pulp Paper Sci. 29(11), pp 386-390.

Koukoulas, A. A. and Jordan, B. D. (1997): Effect of Strong Absorption on the Kubelka-Munk Scattering Coefficient, J. Pulp Paper Sci. 23(5), pp 224-232.

Kubelka, P. and Munk, F. (1931): Ein Beitrag zur Optik der Farbanstriche, Z. Tech. Phys. 11a, pp 593-601.

Kubelka, P. (1948): New Contributions to the Optics of Intensely Light-Scattering Materials. Part I, J. Opt. Soc. Am. 38, pp 448-457.

Kubelka, P. (1954): New Contributions to the Optics of Intensely Light-Scattering Materials. Part II, J. Opt. Soc. Am. 44, pp 330-335.

Moldenius, S. (1983): Light Absorption Coefficient Spectra of Hydrogen Peroxide Bleached Mechanical Pulp, Paperi Puu 65(11), pp 747-756.

Nordman, L., Aaltonen, P. and Makkonen, T. (1966): Relationships Between Mechanical and Optical Properties of Paper Affected by Web Consolidation, Trans. Symp. Consolidation Paper Web, Tech. Sect. Brit. Paper Board Maker's Assoc., London, UK, pp 909-927.

Pauler, N. (2002): Paper Optics, Lorentzen \& Wettre, Kista, Sweden.

Popson, J. S. and Malthouse, D. D. (1996): Measurement and Control of the Optical Properties of Paper, 2nd edition, Technidyne Corporation, USA.

Rundlöf, M. and Bristow, J. A. (1997): A Note Concerning the Interaction Between Light Scattering and Light Absorption in the Application of the KubelkaMunk Equations, J. Pulp Paper Sci. 23(5), pp 220-223.

Schuster, A. (1905): Radiation Trough a Foggy Atmosphere, Astrophys. J. 21, pp 1-22. (Reprinted in Selected Papers on the Transfer of Radiation, Dover, New York, 1966. Edited Menzel, D. H.)

Wick, G. C. (1943): Über ebene Diffusionsprobleme, Z. Phys. 120, pp 702-718.

Manuscript received April 16, 2004 Accepted June, 2004 